\title{
Motion of Conducting Particles Causing Inadvertent Outages in GIS
}

\author{
M. S. Indira and T. S. Ramu \\ Dept of I ligh Yoltage linginecring \\ Indian Institute of Science \\ Bangalore, India
}

\begin{abstract}
Inadvertent failure of a HV gas-insulated system (CIS) is traced to a seemingly innoctous mechanism in particle-induced breakdown. Impending dangers from inconspicuous conducting particles in a large physical system are quite intriguing. The dynamic behavior of the particle due to electric field/particle/fluid medium interaction is a complex phenomenon. To understand this interaction, a credible database has been generated for the field-induced motion of the particles on a physical prototype model. Induced surface charge acquired by the particle is calculated based on the proposed model (field calculations) and by considering it to be a prolate ellipsoid. Methods for deactivating potentially dynamic particles using dielectric coated actipe parts and grounded enclosures, and a novel lechnique called reverse polarity charging have been suggested. Pexformance of GIS in the presence of metallic parlicles has been studied with bare electrodes and with dielectric coatings of different conductivities with a viow to obtain an optimal value of the conductivity.
\end{abstract}

\section{INTRODUCTION}

G As insulated substations or totally enclosed substations have Ibeen a major innowation in power transmission and distribution with proven reliability, and maintenance-free operation. As one is already aware of the attractive features of a gas-insulated system (GIS), they also suffer from certain drawbacks. One of them is outage due to seemingly innocuous conducting particles, which accounts for nearly $50 \%$ of the GIS failures. Probability of broakdown duc to particles is in a region of maximum local field, which may be dofined as the statistical residence time. The field at the particle tip exceeds the liniting diclectric strength of the gas, at least locally, initiating a corona discharge, which in time dewclops into a breakdown. The voltage withstand capability of GIS under normal service conditions due to high concentration of particles depends on numerous interrelated factors such as the length, size, and natire of the particles, posifion of particles with respect to the HV points, dynamics of particles under the electrostatic force and gravitational field, gas pressure, and condifion of the insulator surface. Recent studies have indicated that the GIS can be operated successfully in the presence of particles. Some of these measires are either expensive or posc irreconcilable difficulties in their operation.

In ouder to be able to understand the beharior of particles in an clectric field, dynamic aspects are to be studied. In the simplified model proposed in an earlicr work by the authors [1], the lift-off stresses are determined and hence the induced surface charges. The induced charge is also determined by considering the particle to be a prolate ellipsoid. Hence the lift-off force is estimated which is also an essential parameter in determining the particle dynamics. In order to reduce the adverse of fect of the metallic particles, the earlier literature [2] reports that coated enclosures may be used as a possible method to restrict the motion of the particles. In the present context a novel technique is suggested, which is simple, cost effective and reliable in passivating the particles and thus preventing inadvertent outages.

\section{THEORETICAL}

Certain theoretical aspects of the motion of a particle in an electric field, and its dynamic behavior under the action of various forces aiding and inhibiting the motion of the particle, are considered. Motion of metallic particles in GIS are extensively studied by several authors [3], because particle movement plays a crucial tole in the breakdown of the gaseous medium. These calculations assume, among other simplificatoms, that the net surface charge of the particle remains constant.

\subsection{ESTIMATION OF INDUCED CHARGE ON THE PARTICLE}

A rigorous analysis of the induced surface chatge density $\sigma$ on a particle and its interaction with the electric field in which it is situated, is very complex and often closed-form solutions are not possible. However, an approximate treatmont for calculating $\sigma$ to a fair degree of accuracy is attcmpted. At the outset, a prolate ellipsoidal particle is considcred in a ncarly homogeneous 3-D fied with a possible axial symmotry as shown in Figure 1. The potential at any point between the two electrodes is a function of coordinate axis and the dimension 


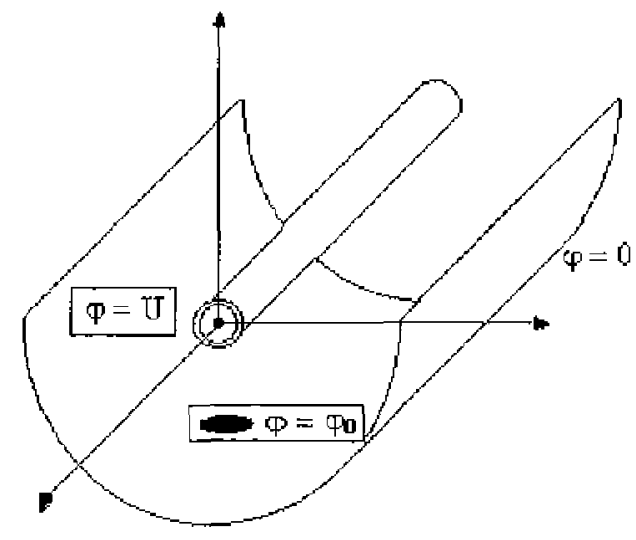

Figure 1. Schematic representation of electrode geomelry and ellipsoidal particle in a $3-D$ coordinate system.

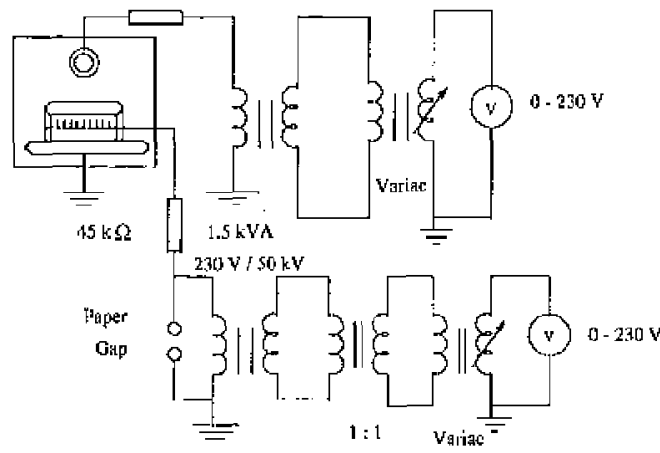

Figure 2. Circuit arrangement for reverse polarity charging

of the ellipsoid. The induced charge on the surface of the conducting ellipsoid can be obtained as in

$$
Q=-8 \pi \varepsilon_{o} A
$$

where $\varepsilon_{0}=8.854 \times 10^{-12} \mathrm{~F} / \mathrm{m}$ is the permittivity of frec space, and in which $A$ is the elliptic integral in 3-D taken over zero and infinity on the variable $\theta$ as

$$
A=-\varphi_{0} \int_{0}^{\infty} \frac{d \theta}{\sqrt{\left(a^{2}+\theta\right)\left(b^{2}+\theta\right)\left(c^{2}+\theta\right)}}
$$

where $a, b$, and $c$ are the scmi-axes of the ellipsoid. In this calculation, the constant $A$ is derived based on the assumption that the potential at the position of particle is the same as in the absence of the particle. The valuc of this integral can be obtained either by numerical integration or referring to a table of olliptic integrals. The surface charge density $\sigma$ is given by

$$
\sigma=\frac{Q}{4 \pi a b c} \frac{1}{\sqrt{\frac{x^{2}}{a^{4}}+\frac{Y^{2}}{b^{4}}+\frac{Z^{2}}{a^{4}}}}
$$

The induced surface charge on the serni-ellipsoidal particle quadtinture to the field, according to Felici, is given by

$$
Q=2 \pi \varepsilon_{o} r l E
$$

where $l$ is the longth of the particle, $r$ the radius of the particle, and $E$ the field seen by the particle.

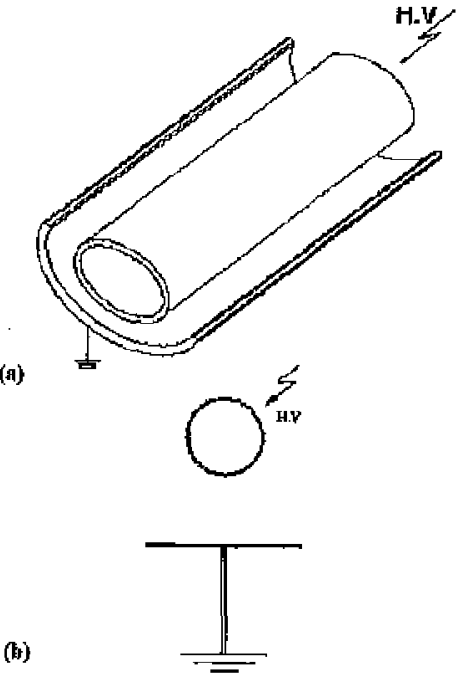

Figure 3. Elcctrode configuration. (a) Eloctrode type 1. (b) Flectrode type 2.

\subsection{THE DYNAMIC ASPECTS OF THE PARTICLE}

The motion of a particle under a time changing ac clectric field is more complex than under direct fields, since the space charge on the particle intertacts with changing fields, In a coaxial system, the partides do not cross the gap unless the electric field is very high and is affected over a number of voltage cycles, after a series of bounces [3]. The maximum altitude attained by the particle is determined by the applied voltage and the loss of charge, due to charge transters, if any, to the modium either by corona or as a pulseless discharge.

\subsection{THE LIFT-OFF FIELD}

This may be defined as the ctitical field at which the particles change their position from rest. It is a function of the initial distribution and orientation of the particles with respect to the electrodes, which dctermines the subsequent particle dynamics. The initial motion at $t_{0}$ is field dependent, and is observed to be independent of the type of gas medium, its pressure, and the length of the particles. Felici suggests approximations for an ellipsoidal particle with its initial position quadrature to the fiold as

$$
\Phi_{r}=\sqrt{\frac{\rho g r}{1.43 \varepsilon_{0}}}
$$

According to $\Lambda$ sano of al. [5], and based on halt-cylinder and fullcylinder geomef'y, the lift-off stress in $\mathrm{kV} / \mathrm{m}$ is given as

and

$$
B_{\mathrm{C}}=808 \sqrt{a \rho}
$$

$$
E_{t}=880 \sqrt{a \rho}
$$

where $a$ is the radius of the particle, and $\rho$ the density of material.

\section{EXPERIMENTAL.}

The stainless steel prototype used to study the particle dynamics for estimating and assessing the lift-off voltages nearly simulated the acthal conditions in a GIS. The chamber was equipped with a Wilson sea! 


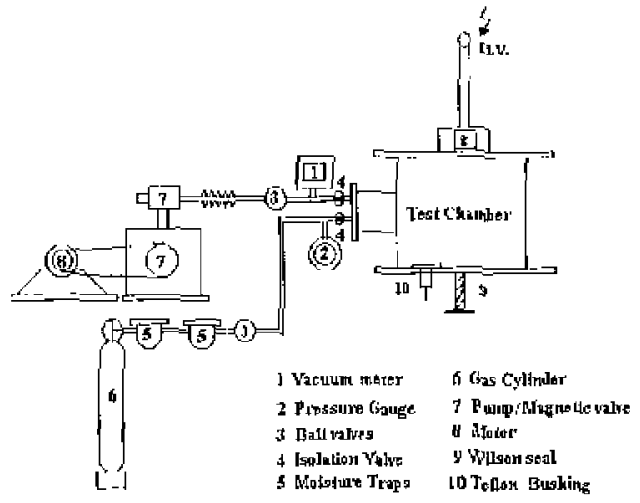

Figure 4, Gas handling systenn.
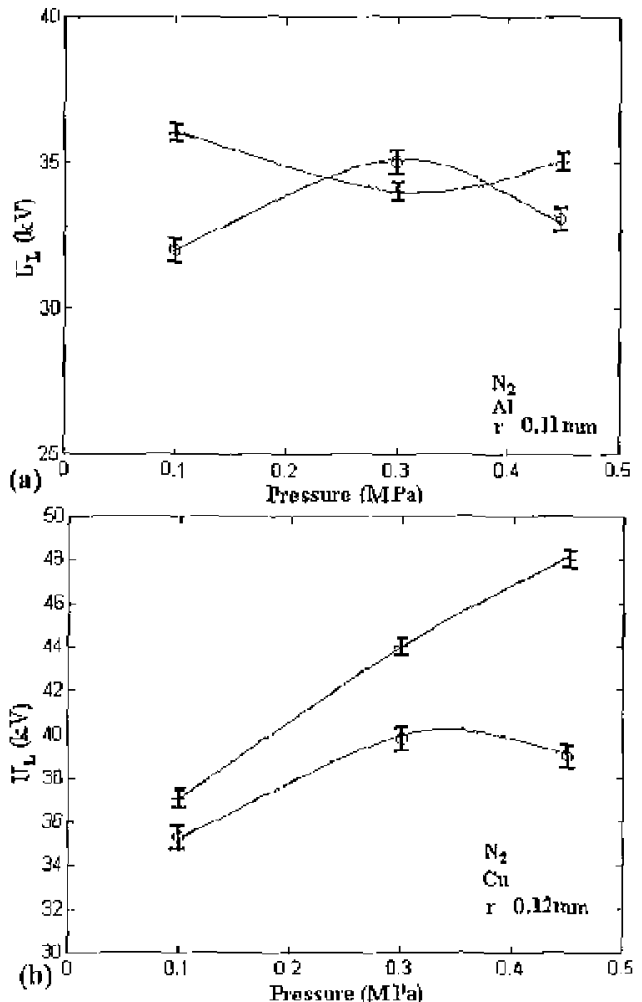

Figure 5 . Measured lift off voltage as a function of pressutte o bare elcetrode type $1,+$ bare electrode iype 2 .

to facilitate the positioning of the particles. Circular ports provided witly toughened glass windows allowed visual observation, illumination and connection for the gas handling system. The controlled pressurized chamber incorporated several additional features such as $\mathrm{HV}$ stress grading of the leadthrough. Spraying of charges of either polarity was achieved by reverse-polarity encrgizing with an endeavor of passivating the partinles. This technique consisted in applying a wolkage proportional to the main I IV supply, but opposing it by $180^{\circ}$ using a scparate auxiliary transformer as shown in Figure 2. Isolation between the two HV sources was obtained by taking the charging power supply
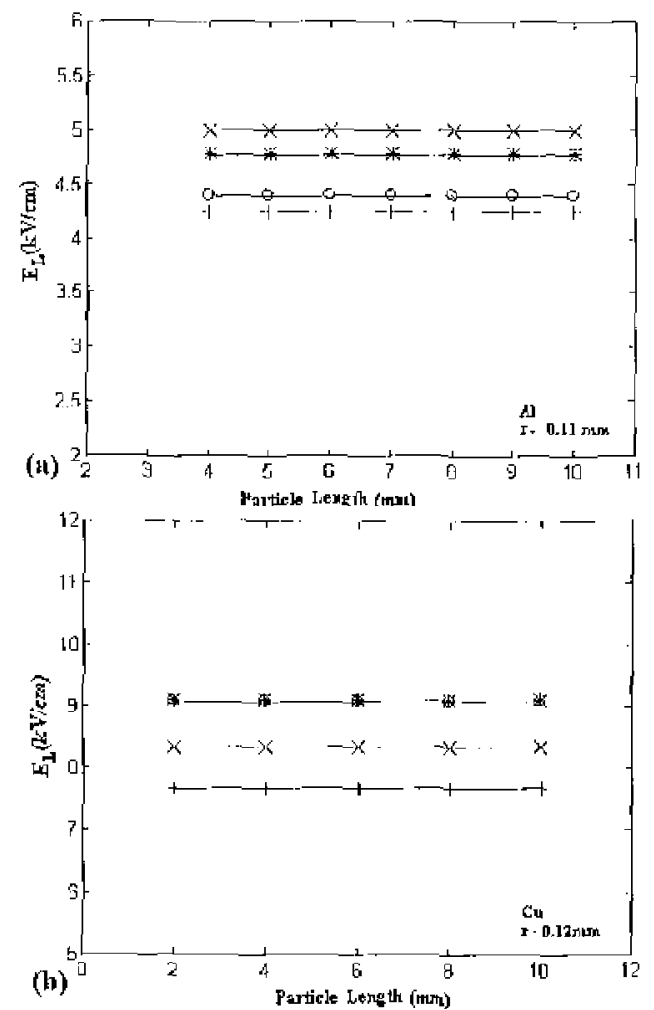

Figure 6. Estimated lift-off stress as a function of particle length. + proposcd model, * Felici's nudel, O Asano-HCM, $\times$ Asano-lCM

through a Tetlon bushing located at the bottom of the chamber.

\subsection{ELECTRODF CONFIGURATION}

Two different electrode geometries, a split-cylinder, thick walled hollow tubular structure and a Rogowski-profiled circular plate thick walled hollow tubular structure, have been considered in the series of experiments reported here. For the sake of cesy identification they arc designated as Type 1 and type 2 electrode systems respectively as shown iu Figure 3. Furthermore these configurations have beon used both as bare and as coatod systems. The electrode sutfaces were highly polished, degreased, and dried before the start of the experiments. A set of geomctrically tormed and polished electrodes werc given a diclectric coating using appropriate dielectric materials such as polyethylene, polypropylene and certain antistatic materials which are obtained by doping certain cpoxy tesins with conducting materials of sclected grain sizes such as pure graphite. The coating thickness waries for different material mentioned here and is in the range of 3 to $20 \mathrm{fm}$.

\subsection{GAS HANDLING SYSTEM}

Figure 4 shows details of the gas handhing system. The prototype of the Gis chamber was equipped with pressure vaculm valves and gauges to monitor the degree of prior evacuation and final pressurc. The chamber was evacuated to a pressure of $1 \mathrm{mPa}$ before filling with the gas medium. Pressutes were thaintained between $0: 1$ to $0.5 \mathrm{MPa}$. The gas was passed through moisture traps $\left(\mathrm{CaCl}_{2}\right.$ and silica gel) with 

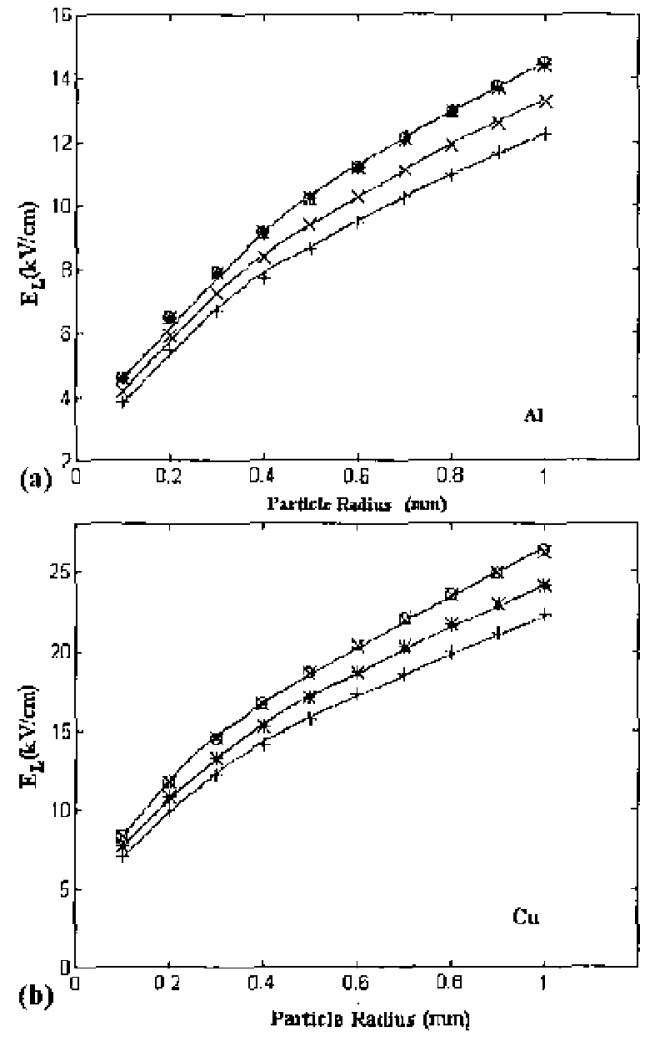

Figure 7. Estimated lift-off stress as a function of particle size. (a) A] particle, (b) Ct particle. o leelici's modd, + proposed model, * Asano$\mathrm{HICM}, \times$ Asano-rCM.

a regulated flow rate. The evacuation and the gas inlot systems were isolated using high quality ball walves. Safety valves wore incorporated to let out excess gas when necessary.

\subsection{TEST AND MEASURING EQUIPMENT}

High ac voltage at power frequency was generated by an HV cascaded measuring (test) transformer rated for $10 \mathrm{kVA}, 220 \mathrm{~V} / 110 \mathrm{kV}$ (GE). The system was observed to be partial discharge (PD) free up to $1.2 \times$ the rated voltage. The low voltage supply to the transformer came from a regulator rated for $60 \mathrm{~A}, 240 \mathrm{~V}$, and $50 \mathrm{~Hz}$. A current limiting resistor, also $P^{\prime} \mathrm{D}$ free, with a resistance of $40 \mathrm{M} \Omega$ provided the rcquired curtent protection. An auxiliary voltage transformer rated for $1.5 \mathrm{kVA}$, $230 \mathrm{~V} / 50 \mathrm{kV}$ along with a $25 \mathrm{kVA}, 110 \mathrm{~A}, 230 \mathrm{~V}$ variac and a $45 \mathrm{k \Omega}$ current limiting resistor were used in the experiments to study the passivating effects of reverse polarity charging. The necessary phase shift in the voltago waveform was dorived from an intermediate 1:1 isolation transformer. Voltage across the specimen was obtained by subtracting rectorially the voltage drop across the current limiting resistor from the source voltage.

Measurement of resistivity helps in predicting the ability of the insulating material to dissipate the buildup of electrostatic charge. Materiats that are coated or chemically treated to contain an internal antistatic agent have static dissipative chatacteristics that ate a function of surface
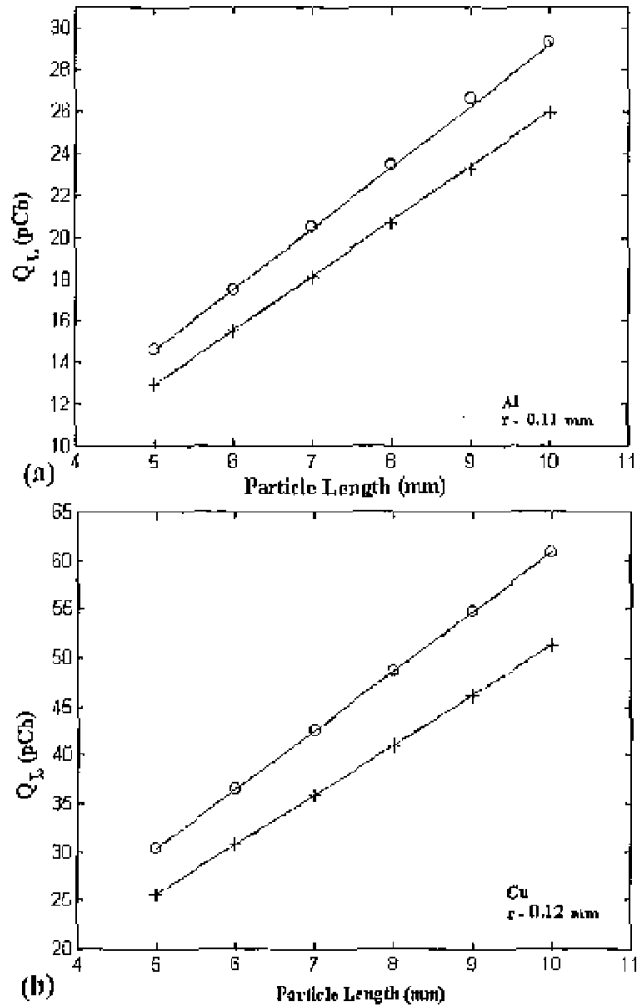

Figure 8. Calculated induced surface charge $Q_{l}$, at lift-cff o Felicit's model. + proposed model.

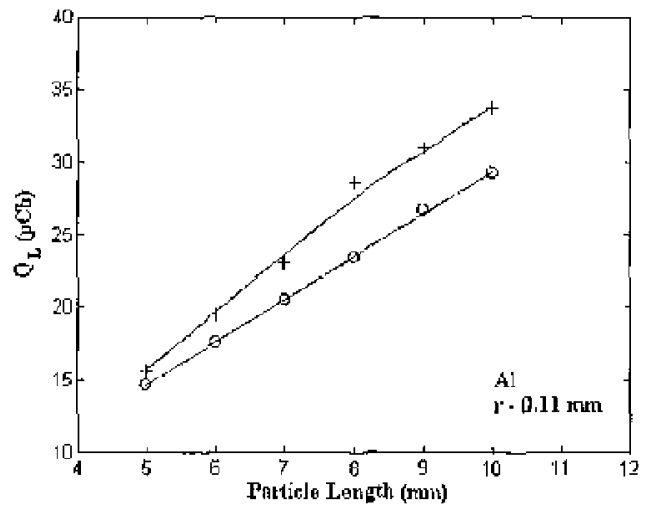

Figure 9 . Calctlated induced surface charge $Q$, at lift-off o Pelici's model, + proposed modcl.

resistivity. $A$ surface/voltage resistivity probe Modcl 803 A measured the resistivity of the coating materials. The desigu of the model probe alectrode configuration was derived from the applicable formulae mentioned in ANSI/ASTM D257 [6].

In the series of experiments roported here, filamentary particles of aluminum and copper were chosen, since they arc more common in Gis and ate very critical to the system performance. No attempt was made to smoothen the wire ends in order to simulate the actual condition in a GIS. 

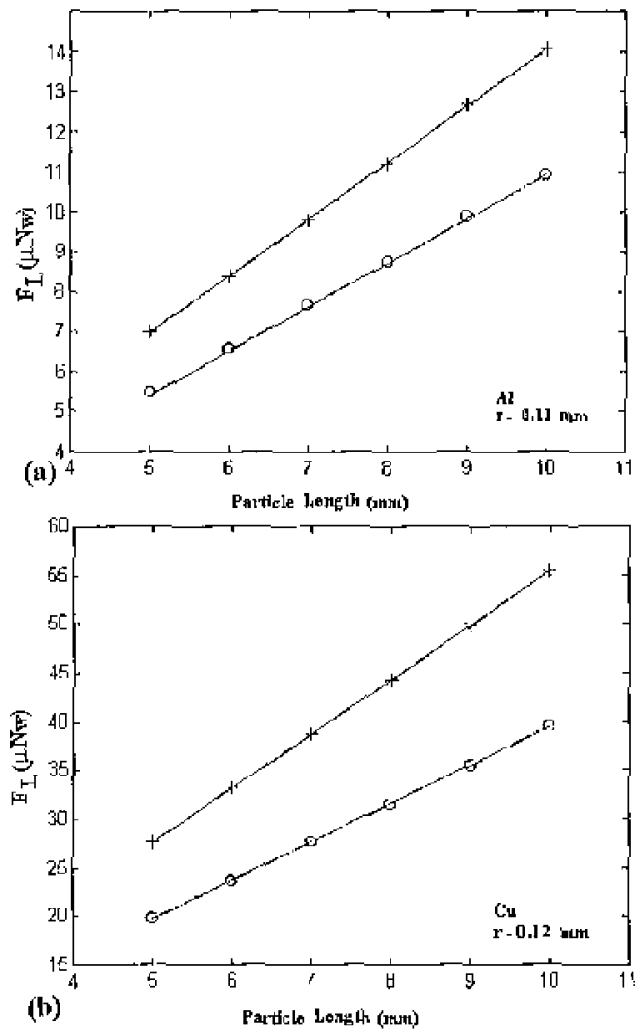

Figure 10, Estimated elchtostatic force at lift-off as a function of particle length a Felici's model, + proposed model.

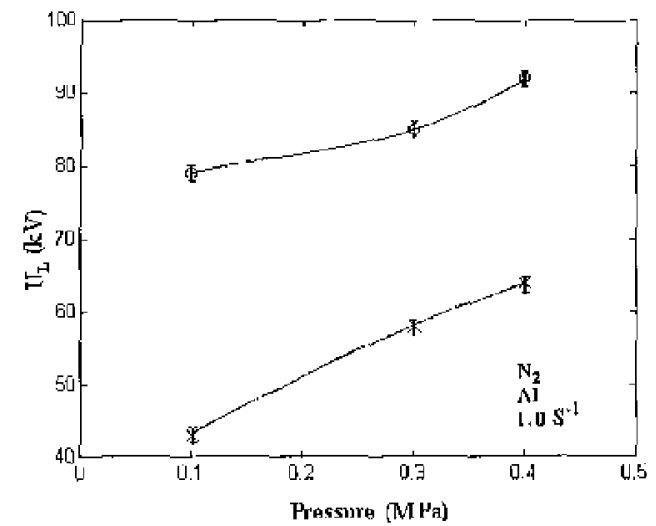

Figure 11. Measured lift-off vollage as a function of pressure, + dielectric coated grounded enclusure, o both elcctrodes dielectric coated.

\section{RESULTS AND DISCUSSIONS}

Particles in practical systems have a wide vatiety of sizes, shapes and are materials of differing conductivities and densities. Many of these factors influence the particle charging and charge leakage mechatisms, as do the dielectric properties of the gas and its pressure. Concomitantly, these factors also have a significant influence on the motion of the particles and the degree to which they migrate into the high field zonc.
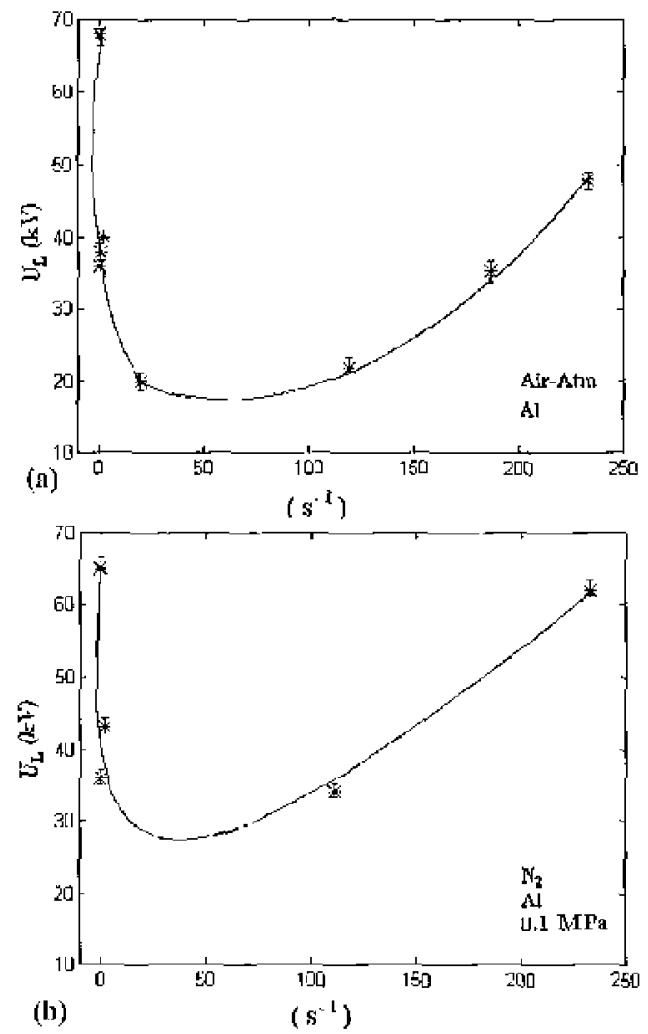

Figure 12. Measured lift-cff voltage as a lunction of conductivity of the coating material. (a) $\Lambda \mathrm{ir}$, (b) $\mathrm{N}_{2}$.

\subsection{RESULTS}

The lift-off voltages as a function of pressunc for aluminum and copper particles for the two clectrode systems are depicted in Figures 5(a) and (b). The liftroff voltage seems to be independent of the electrode system. The lift-off stresses obtained from the proposed model [1] are compared with Felicl [4] and Asano et al. [5], and for both aluminum. and copper particles are shown in Figures $6(a)$ half cylinder mode: (HCM) and (b) full cylinder model (FCM). Figures 7(a) and (b) depict the variation of the lift-off voltage with the size of the particle. The obvious observation is that the lift-off voltage does not depend on the particle length and pressure of the medium but depends on the particle material density and the size.

It may be observed that the plotting positions have been indicafed with the mean value centered atound one siandard deviation on the mean value, assuming that the data at any one voltage have a Gaussian scattcr. This procedure of plotting is used in Figures 5 and 11 to 14 . Actually, on the graphs such scatter does not appear to be very significant.

The induced surface charge accuired by the particle as a function. of length at lift-off based on the proposed model and Folici's formula has been compared. [igures $8(a)$ and (b) amply support agreement betwen the two modes of field calculation. The induced surface charge calculated by considering the particle to be prolate ellipsoid, compares woll with the model based on Felici's calculation as shown in Figure 9. The clectrostatic force cxperienced at lift-off as a function of length for 


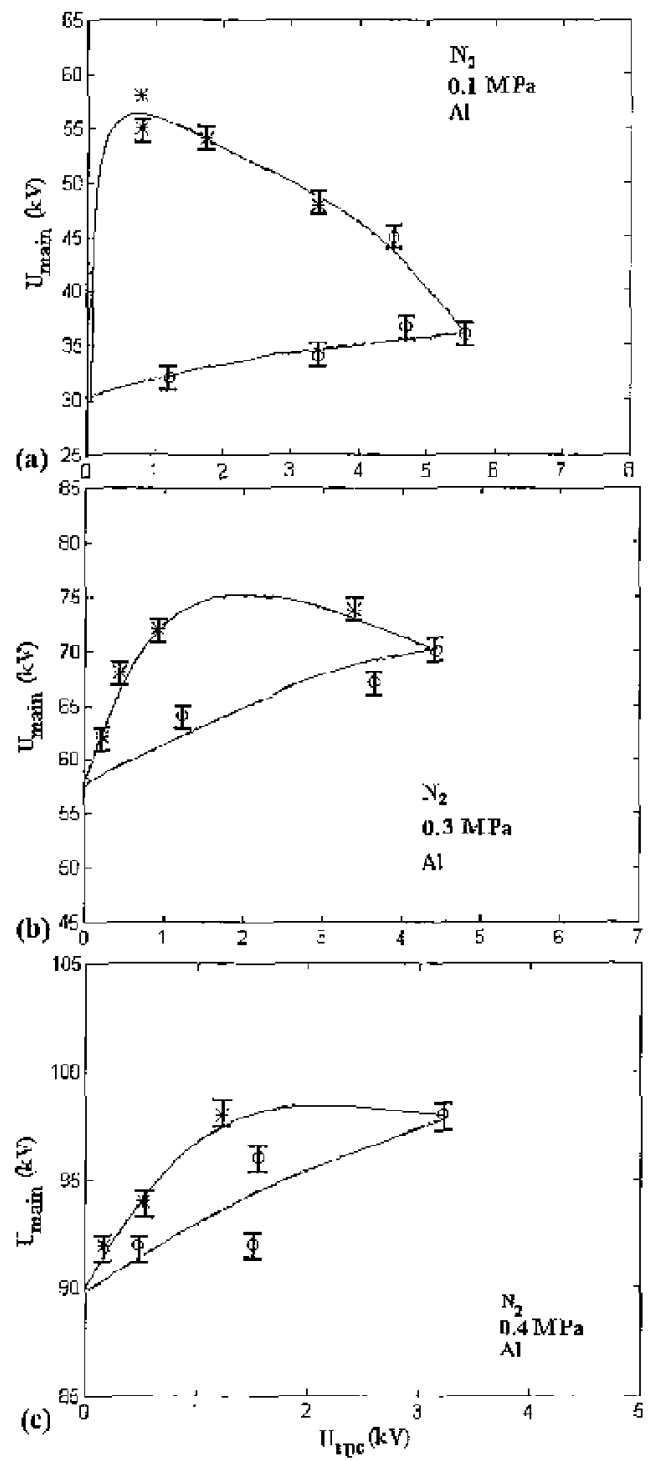

Figure 13. Hysleresis effect with bare electrodes. (a) $0.1 \mathrm{MPa}$, (b) $0.3 \mathrm{MPa},(c) 0.4 \mathrm{MPa}$

a given size of the particle is estimated and given in Figures 10(a) and (b).

The dielectric coating of a metallic surface affects the particle charging mechanism. Coating the endosure increases the lift-off voltage considerably. Various dielectric materials have been tried by the authors [1]. Figure 11 suggests that there is considerable improvement in the performance, by having both electrodes coated rather than with only coated enclosure or with bare electrodes. A critical minimum has been observed with contsiderable repoatability in both atr and nitrogen, as depicted in Figures 12(a) and (b), a plot of the variations of $O_{L}$ with conductivity of the coating materials. Similar behavior has been observed in nitrogen at pressures of 0.3 and $0.4 \mathrm{MPa}$.

In an effort to mitigate the probiem of particle charging and the en-

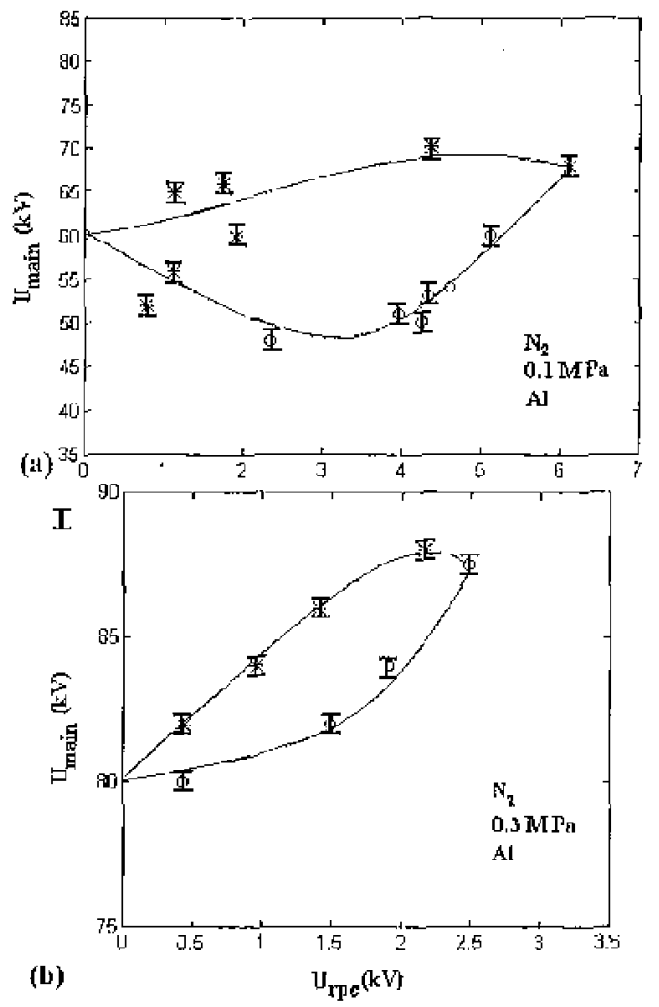

Figure 14. Iiysteresis effect with coatcd elcetrodes. (a) $0.1 \mathrm{MPa}_{\text {, (b) }}$ $0.3 \mathrm{MPa}$.

suing breakdown, a new method of stripping the charge was tried out on both bare and coated electrodes at different pressures of the gas. $A$ plot of the applied woltage $U_{m}$ as a function of reverse polarity charging voltage $U_{r}$ at different pressures are shown in Figures 13(a), (b) and (c) exhibited a nearly hysteresis (closed two-loop curves) behavior. The area enclosed could be related to the charge and hence the energy accumulated by the particle.

\subsection{DISCUSSION}

The caiculation of the actual field seen by the particle is quite complex. In this work an approximation has ben made in which the particles are treated as ellipsoids and in the limit the coordinates are made small enough so as to make them thin strip of wires. The fields seen by the particle in such an event is estimated to be 2 to $5 \times$ the actual field at that point.

The empirical relations suggested by Folici [4] and Asano el a]. [5] can be used to evaluate the surface charge accumulated by the particle. The small differences in the charges calculated using the above nontioned relations and the proposed model is believed to be due to the approximation to the ficld suggested above.

In the literature $[7,8]$ one of the methods suggested for mitigating particle activity is to use dielectric coated electrode. By using suitable coatings, mentioned carlier, the micro-projections are smoothened, reducing the bounce heights and thus restricting the particle movement. It may be seen that the concept of electrical stress grading iu cables 


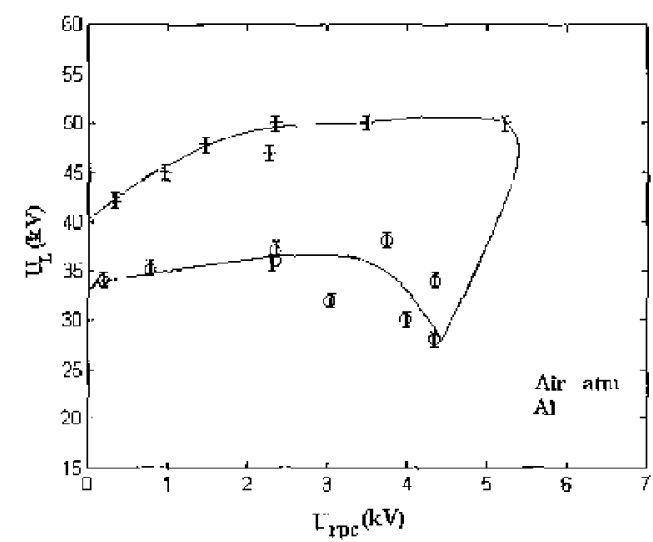

Figure 15. Hysteresis effect with bare electrodes (air).

and generator stator bars could be involved in GIS by the use of semiconducting coating. If the coating is treated as a lumped cquivalent $R C$ circuit, with a time constant larger than corresponds to the power frequency, then the charge acquired by the particle will be smaller than the required charge for liff-off and hence rosults in an increase in the lift-off voltage levels. It is also seen that conting both the HV electrode and the grounded enclosure enhances the performance of the system.

It has been reported in [ 9 that the mechanism of charging of particles is due to l'D micro-discharges at low pressure and high fields. With alternating voltages, charges were found to accumulate in the negative half cycle and discharge in positive half cycle. The charge quantity $Q_{v_{k}}$ of the PD in the negative cycle was found to bo larger that $Q_{p}$ in the positive half cycle, at Low pressures, in particular. The difference $\left(Q_{r_{L}}-Q_{p}\right)$ of the charges generated every cycle rosides on the particle until the total charge reaches the throshold value for lifting.

In the light of the abowe discussion, the authors proposed a new method of charge stripping. This may be allod the "reverse polarity charging. The plysical basis of this method is to use charged particles of polarity opposite to that acquired by the particles with the aid of the setup shown in Figure 2. As has been mentioned carlier, a plot of $U_{m}$ and $U_{r}$ appears to show a hysteresis behavior. In this method of chargitig, the accutmulated charge reducos with pressure in both bare and coated electrodes as shown in Figures 13(a), (b), and (c) and Figures 14 (a) and (b).

A similar effect can be observed when bare and coated electrodes are compared at a constant working pressure, Figures $13(a)$ and 14(a) and 13(b) and 14(b). Thus the particles require higher lift-off voltages to reach the threshold value of the charge, because the area cnclosed by the loop can be shown to be proportional to the charge and hence the energy of the particlc. A similar lysteresis behavior is exhibited with air at atmospheric pressure as shown in Figure 15. Hysteresis under similar experimental conditions is observed with copper particles also.

\section{CONCLUSIONS}

TN sunmary, the measured lift-off voltages seem to be independent of the electrodo systems in question. The induced surface charge on the particle using prolate ellipsoidal approximation developed and based on the proposcd model compares well with existing models. The lift-off forces astimated by the proposed model are in good comparison with those obtained with Felici's formula and the available data. The lift-off voltage levels arc accentuated considerably when both electrodes are coated with a dielectric, in comparison to only the enclosure being coated, and to the case of bare electrodes. The reverse polarity charging method has a considerable adwantage over the dielectric conting method in that the particles are passivated to much higher woltage lovels. A similar effect is observed with coated enclosures also with the new technique. Better performance could be expected if the technique is used in conjunction with a conted enclosure. The area of the hystetcsis loop is proportional to the charge and hence the energy, successively diminishes with pressure with both bare and coated enclosures, requiring higher lift-off voltages.

\section{REFERENCES}

[1] M. S, Indiria and T. S, Randu, "Theoretica] ant Expetimontal Model for larlicle Ini-

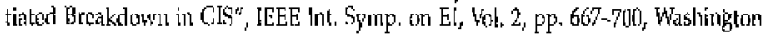
DC., USA, 1995

[2] K. S. Prakash, K. D. Srivatsava and M. M. Marcos, Morethent Of Wire Paticles in Compressed $\mathrm{SF}_{5}$ GIS upon Dietectric Coal hig of Flectrodes", IEEE 7rans. DFI, Wol. t. Pp. 344-317, 1997 .

[3] Wostinghouse Research, Development Center, Pittsburgh and Peninsylwania, "Tr-

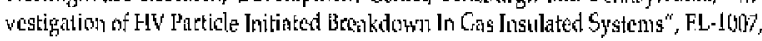

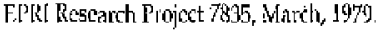

[4] N. J. Felici, "Forces at Charges de Petils Objects en Contad Avee une Plitectrode

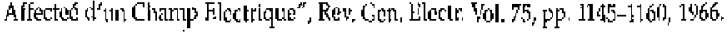

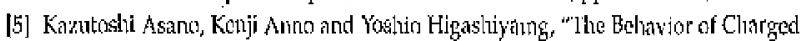
conducting partinles in. Electric Fidkls", ITEE Trans. On Endusty Applications, Val. 33, No. 3, May-Junce, 1997.

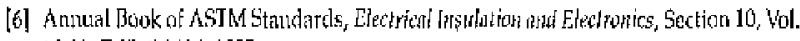
10.01, D69-D2484, 1995.

[7] D. Chee-f line and. K. D. Scivatsava, "Instlation Pestormance of Diclectric Coatcd Filoctrodes in Sulfurhexafluerido Gas", IEEE 'Irins. EI, Vol, 10, no. 4, pp. 119-124, 1975 .

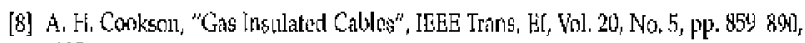
1985.

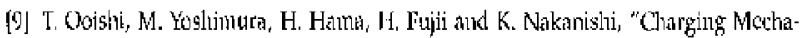
nisms of a Conctucting Particle on Dielect ric Conted at ac and DC Electric Firelds"

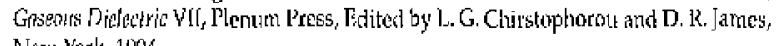
New York, 1994.

Manuscripi was received on 16 Novembor 1998, in final form 7 September 1999. 